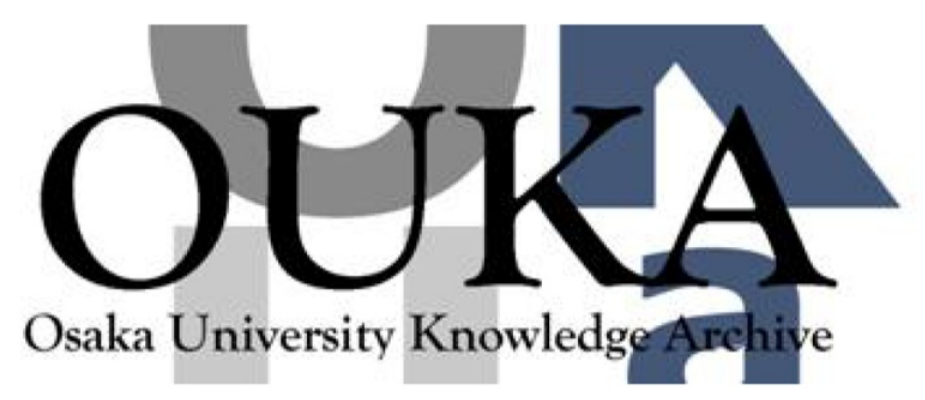

\begin{tabular}{|c|l|}
\hline Title & $\begin{array}{l}\text { Time-resolved study of luminescence in highly } \\
\text { luminescent disubstituted polyacetylene and its } \\
\text { blend with poorly luminescent monosubstituted } \\
\text { polyacetylene }\end{array}$ \\
\hline Author(s) & $\begin{array}{l}\text { Hidayat, Rahmat; Tatsuhara, Satoshi; Kim, Dong } \\
\text { Wook et al. }\end{array}$ \\
\hline Citation & $\begin{array}{l}\text { Physical Review B - Condensed Matter and } \\
\text { Materials Physics. 61(15) p. 10167-p. 10173 }\end{array}$ \\
\hline Issue Date & $2000-04-15$ \\
\hline oaire:version & VoR \\
\hline URL & https://hdl.handle. net/11094/75858 \\
\hline rights & $\begin{array}{l}\text { Copyright (2000) by the American Physical } \\
\text { Society }\end{array}$ \\
\hline Note & \\
\hline
\end{tabular}

Osaka University Knowledge Archive : OUKA

https://ir. Library. osaka-u. ac. jp/

Osaka University 


\title{
Time-resolved study of luminescence in highly luminescent disubstituted polyacetylene and its blend with poorly luminescent monosubstituted polyacetylene
}

\author{
Rahmat Hidayat, Satoshi Tatsuhara, Dong Wook Kim, Masanori Ozaki, and Katsumi Yoshino \\ Department of Electronic Engineering, Faculty of Engineering, Osaka University, 2-1 Yamada-oka, \\ Suita, Osaka 565-0871, Japan \\ Masahiro Teraguchi and Toshio Masuda \\ Division of Polymer Chemistry, Faculty of Engineering, Kyoto University, Yoshida-Honmachi, Sakyo-ku, Kyoto 606-8317, Japan
}

(Received 26 February 1999; revised manuscript received 2 June 1999)

\begin{abstract}
A highly luminescent disubstituted polyacetylene, poly(1-phenyl-2- $p$ - $n$-butylphenylacetylene) (PDPA- $n \mathrm{Bu}$ ), and its blend with a poorly luminescent monosubstituted polyacetylene, poly(1$o$-trimethylsilylphenylacetylene) (PPA- $o \mathrm{SiMe}_{3}$ ), are studied by time-resolved photoluminescence (PL) spectroscopy. In pure PDPA- $n \mathrm{Bu}, \mathrm{PL}$ intensity at short wavelength decays faster than that ang wavelength, whereas PL spectra exhibit a dynamic Stokes shift to longer wavelengths with time. In blends of PDPA$n \mathrm{Bu} / \mathrm{PPA}-o \mathrm{SiMe}_{3}$, only PL originating from PDPA- $n \mathrm{Bu}$ is observed, without contribution from PPA- $o \mathrm{SiMe}_{3}$. The PL lifetime drastically decreases upon mixing a small amount of PPA- $o \mathrm{SiMe}_{3}$ in PDPA- $n \mathrm{Bu}$. The PL characteristics of pure PDPA- $n \mathrm{Bu}$ and its blend with PPA- $o \mathrm{SiMe}_{3}$ are discussed in terms of lattice/vibrational relaxation of the excitonic state and exciton migration.
\end{abstract}

\section{INTRODUCTION}

Conjugated polymers have attracted much attention since quasiparticles such as solitons, polarons, and bipolarons, which can be created by doping, photoexcitation, and electron (or hole) injection, were found to play important roles as free charge carriers in these materials. ${ }^{1}$ Various phenomena unique to these materials have been successfully explained using the concept of these quasiparticles, and many practical applications have been proposed. Conjugated polymers with highly extended $\pi$-electron systems in the main chains are also called conducting polymers because they exhibit metallic properties in doped states. Conjugated polymers used as luminescent materials have been intensively studied after Burroughes $\mathrm{et} \mathrm{al.} .^{2}$ demonstrated electroluminescence (EL) in poly ( $p$-phenylenevinylene) (PPV), followed by other groups ${ }^{3,4}$ employing other polymers.

In general, it has been accepted that photoluminescence (PL) in conjugated polymers originates from radiative recombination of a singlet exciton, which is a direct product of intrachain photoexcitation. The exciton is allowed to relax to a lower energy prior to radiative recombination, through a molecular conformational change/lattice relaxation or exciton migration to a longer conjugation segment either on the same chain or on neighboring chains, which shifts PL spectrum to longer wavelengths with time. ${ }^{5,6}$ In several conjugated polymers, PL spectra obtained from film, solution, and solid solution of polymers can be different. These results have been attributed to luminescence from interchain processes, due to strong interchain interactions, such as interchain excitons or excimers ${ }^{7,8}$ (pairs in the excited state) and aggregate states $^{9}$ (pairs in the ground state which can be directly optically excited).

The luminescent conjugated polymers so far studied are mostly polymers with a nondegenerate ground state, such as PPV and polythiophene (PT) derivatives. On the other hand,
PL studies of polymers with a degenerate ground state, that is, polyacetylene and its derivatives, have rarely been reported. In the early stage of study, trans-polyacetylene was concluded to be a nonluminescent polymer due to fast relaxation of the photoexcited state into soliton pairs because of strong electron-phonon interaction. ${ }^{10}$ However, even in trans-polyacetylene, weak PL was reported to be observed in the infrared region, and this has been explained in terms of the relative energy level of the lowest excited states, $1{ }^{1} B_{u}$ and $2{ }^{1} A_{g}$ excited states, ${ }^{11,12}$ but is not related to the recombination of soliton pairs. That is, in general, strong PL can be expected when the $1^{1} B_{u}$ excited state is located at a lower energy than the excited state $2{ }^{1} A_{g}$. Photoexcitation studies of trans-polyacetylene and a poorly luminescent monosubstituted polyacetylene, poly(1-o-trimethylsilylphenylacetylene) (PPA-oSiMe ${ }_{3}$ ), indicated that the $2{ }^{1} A_{g}$ excited state lies below the $1{ }^{1} B_{u}$ excited state. ${ }^{13,14}$ Several theoretical studies on the electronic structure in finite and infinite polyenes (i.e., trans-polyacetylene) with strong electron correlations showed that the lowest excited state is $2{ }^{1} A_{g}$, which is associated with either triplet-triplet pairs or soliton pairs. ${ }^{15,16}$ Therefore, it can be expected that the $2{ }^{1} A_{g}$ state is the relaxation channel that competes with the $1{ }^{\mathrm{f}} B_{u}$ channel, depending on the relative energy level between them.

Since PL is related to the nature of the lowest excited states, it should also be possible to obtain strong luminescence even in polymers with a degenerate ground state. Recently, intense PL and EL have been reported in substituted polyacetylenes, that is, poly(diphenylacetylene) (PDPA) derivatives and poly(alkylphenylacetylene) (PAPA). ${ }^{17}$ In general, the introduction of side chains could lift the groundstate degeneracy. However, the characteristic of the groundstate degeneracy in one of PDPA derivatives, that is, poly(1phenyl-2- $p$ - $n$-butylphenylacetylene) (PDPA- $n \mathrm{Bu}$ ), has been recently confirmed by Gontia et al. through the observation 


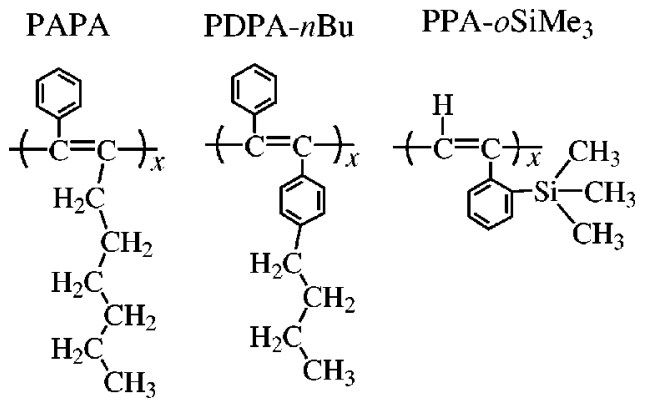

FIG. 1. The molecular structures of PAPA, PDPA- $n \mathrm{Bu}$, and PPA- $o \mathrm{SiMe}_{3}$.

of soliton formation. ${ }^{18}$ The soliton formation was identified by the appearance of midgap band in doping-induced absorption spectra and in steady-state and transient photoinduced absorption spectra, but the quantum efficiency of the formation is much lower than PL quantum efficiency, which was explained in terms of the possibility of ordering the excited state as mentioned above.

In contrast to other luminescent conjugated polymers, these substituted polyacetylenes do not show large difference in spectral shape between the steady-state PL spectra of the solid film, solution and solid solution, suggesting strong exciton confinement and weak interchain interaction. The luminescence characteristics and efficiencies have been found to depend on the molecular structure of side chains, suggesting different exciton confinement strength or different competitive rate-determining processes such as nonradiative decay or trapping by charge carriers/defects. ${ }^{19,20}$ However, exciton dynamics in these polymers have not been investigated in detail so far.

In this paper, we report time-resolved PL spectroscopy applied for studying the exciton dynamics in these substituted polyacetylenes. In this work, we use PDPA- $n \mathrm{Bu}$, which has a band gap of about $2.7 \mathrm{eV}$ and exhibits intense green PL peaking at $2.3 \mathrm{eV}$ or Stokes-shifted by about $0.4 \mathrm{eV}$ from the absorption spectrum edge. The present experimental results show the dependence of PL decay characteristics on the emission wavelength and the dynamic Stokes shift to a longer wavelength, which reflects the dynamics of exciton relaxation to a lower energy state. For further study of the exciton dynamics in this polymer, we use polymer blends of PDPA- $n \mathrm{Bu}$ with poorly luminescent PPA- $o \mathrm{SiMe}_{3}$, because in the previous study we observed that steady-state PL of PDPA- $n \mathrm{Bu}$ was significantly quenched when mixed with even a small amount of PPA-oSiMe ${ }_{3}{ }^{21} \mathrm{PPA}^{2} o \mathrm{SiMe}_{3}$ was considered to exhibit negligible intensity of PL, but weak EL was observed upon voltage application in the red spectral range.

\section{EXPERIMENT}

The synthesis of PDPA- $n \mathrm{Bu}, \mathrm{PAPA}$, and PPA- $o \mathrm{SiMe}_{3}$, whose molecular structures are shown in Fig. 1, has been described elsewhere. ${ }^{22,23}$ Thin films of polymers were prepared by spin coating the polymer in chloroform solution onto quartz substrates. For preparing polymer blend films, PDPA- $n \mathrm{Bu}$ and PPA- $o \mathrm{SiMe}_{3}$ were mixed at various molar ratios (the ratios of the number of moles of PPA- $O \mathrm{SiMe}_{3}$ to that of PDPA- $n \mathrm{Bu}$ ) in chloroform and then spin coated.

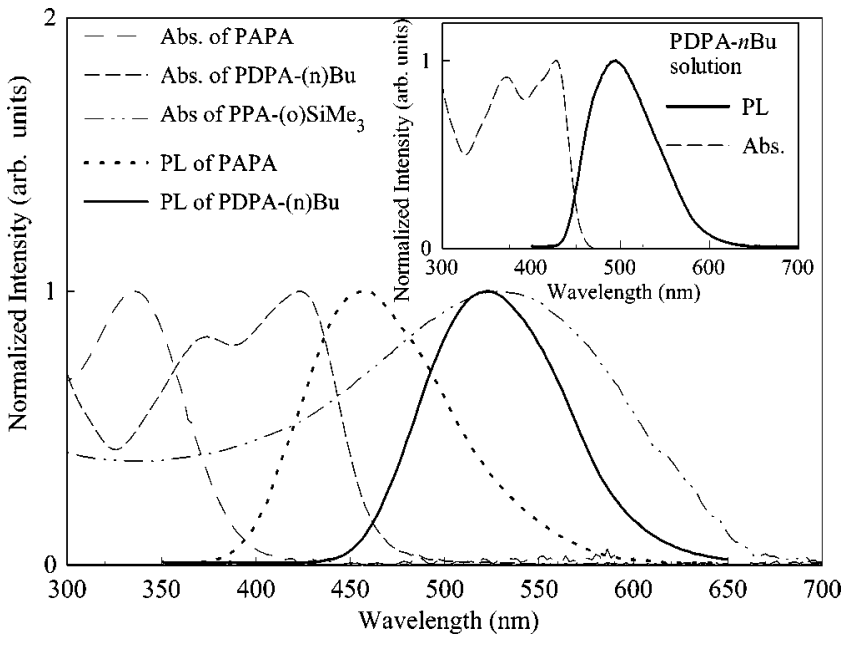

FIG. 2. The absorption and steady-state PL spectra of PDPA- $n \mathrm{Bu}$, PAPA, and PPA- $o \mathrm{SiMe}_{3}$. Inset: The spectra of PDPA- $n$ Bu solution in chloroform.

Absorption and steady-state PL spectra of the spin-coated film were measured in vacuum using a spectrophotometer (HP8452 or Hitachi 330) and a fluorescence spectrophotometer (Hitachi F-2000), respectively. Time-resolved PL measurements were carried out using a femtosecond (fs) laser system. That is, light from a cw-diode-pumped laser (Millenia, Spectra Physics) was passed through a Ti:sapphire mode-locked laser (Tsunami, Spectra Physics) to obtain a laser pulse of $80 \mathrm{fs}$ with pulse trains of $82 \mathrm{MHz}$, and then frequency doubled to $400 \mathrm{~nm}$ by a second-harmonicgeneration unit utilizing beta-barium-borate crystals (Frequency Doubler 3985, Spectra Physics). The laser beam has a spot area of about $4 \mathrm{~mm}^{2}$ and energy of about $1 \mathrm{pJ} /$ pulse. The PL decay and time-resolved PL spectra were measured simultaneously using a streak scope camera (Streak Scope C4334, Hamamatsu). The time resolution of this experiment was limited by the time resolution of about $15 \mathrm{ps}$ of the streak scope camera. The sample was set in a vacuum chamber with quartz windows and all measurements were carried out in vacuum at room temperature. The PL quantum efficiency measurements were performed using the method described by Greenham et al., ${ }^{24}$ that is, by utilizing an integrating sphere for collecting the photons and an argon-ion laser with a wavelength of $360 \mathrm{~nm}$ as the light excitation source.

\section{RESULTS AND DISCUSSIONS}

Figure 2 shows the absorption and steady-state PL spectra of PDPA- $n \mathrm{Bu}, \mathrm{PAPA}$, and PPA- $o \mathrm{SiMe}_{3}$ films, whereas the inset figure shows the same spectra for $\mathrm{PDPA}-n \mathrm{Bu}$ in choloroform solution. Disubstituted polyacetylenes exhibit intense PL with a large Stokes shift: green PL with the Stokes shift ranged from 0.3 to $0.4 \mathrm{eV}$ for PDPA derivatives and blue PL with the Stokes shift of about $0.6 \mathrm{eV}$ for PAPA. In contrast, PL of PPA- $o \mathrm{SiMe}_{3}$ could not be detected by the same spectrophotometer with that used to measure the steady-state PL spectra of PDPA- $n \mathrm{Bu}$ and PAPA. Therefore, monosubstituted polyacetylene is considered to exhibit negligible PL.

Although the molecular structure of PDPA- $n$ Bu can be regarded to contain stilbene units, we interpret the observed 
PL as originating from the main chain, but not from stilbene units. We have studied PL in several PDPA derivatives with different substituent molecules attached to the phenyl rings. The PL intensity was found to depend on the substituents, although the absorption spectra did not appreciably change. Relative to PDPA- $n \mathrm{Bu}$, the PL intensity became weaker when the substituent was trimethylsilyl, but stronger when the substituent was an alkyl chain longer than butyl. ${ }^{20}$ If PL originates from stilbene units that are influenced by the main-chain conjugation environment, the introduction of different substituents should not only change the PL intensity but also the PL spectra, contrary to the experimental results. That is, even in PDPA derivatives with substituents of a much longer conjugation than stilbene, the PL spectra did not appreciably change. Due to a steric effect induced by the large size of side chains (phenyl rings and/or substituent groups), the phenyl rings may not be coplanar with the main chain so that the main-chain conjugation may be separated from the phenyl ring conjugation.

The introduction of side chains could induce several effects such as interchain separation and main-chain bond twisting, which can confine excitons in the main-chain segments. It could also introduce conformational defects on the main chain, which might quench the PL. Different side-chain molecules could induce such effects with different strengths, resulting in a difference in PL intensity. From the results of PL quantum efficiency measurements, it was found that the PL quantum yield of PDPA- $n \mathrm{Bu}$ is about $45 \%$ whereas the PL quantum yield of PPA-o $o$ SiMe 3 is less than $1 \%$. The PL quantum yield of PAPA is inferred to be higher than that of PDPA- $n$ Bu because PAPA exhibits stronger PL. These facts are also consistent with the interpretation that the observed $\mathrm{PL}$ is originated from the conjugated main chain but not from stilbene moieties, because if PL is originated from stilbene the PL quantum efficiency of PDPA- $n$ Bu that contains stilbene units should be higher than that of PAPA that contains asymmetric side-chain pairs, contrary to the observation.

For further clarification, we measured the PL spectra of a stretched PDPA- $n$ Bu film. The PL optically polarized parallel to the stretching direction was stronger than PL polarized perpendicular to it. The anisotropy in PL indicates that PL preferentially occurs parallel to the main chain, suggesting that PL originates from the main-chain segments.

Here, as a representative of disubstituted polyacetylenes for the time-resolved PL study, pure PDPA- $n \mathrm{Bu}$ and its blend with PPA-oSiMe 3 were studied. Figure 3 shows the PL decay of a pure PDPA- $n$ Bu film observed at three different PL wavelengths of 475,550 , and $625 \mathrm{~nm}$ within a time window of $5 \mathrm{~ns}$. The duration of the excitation laser pulse of about 80 fs is considerably short compared to the time window of observation; therefore, the observed decay curve is due to PL decay without convolution of the laser pulse. The PL decay at the wavelength of $625 \mathrm{~nm}$ can be well approximated by single-exponential decay:

$$
I(t)=I_{0} \exp (-t / \tau),
$$

where $I(t)$ is the PL intensity at time $t, I_{0}$ is the initial intensity, and $\tau$ is the lifetime constant, which is evaluated to

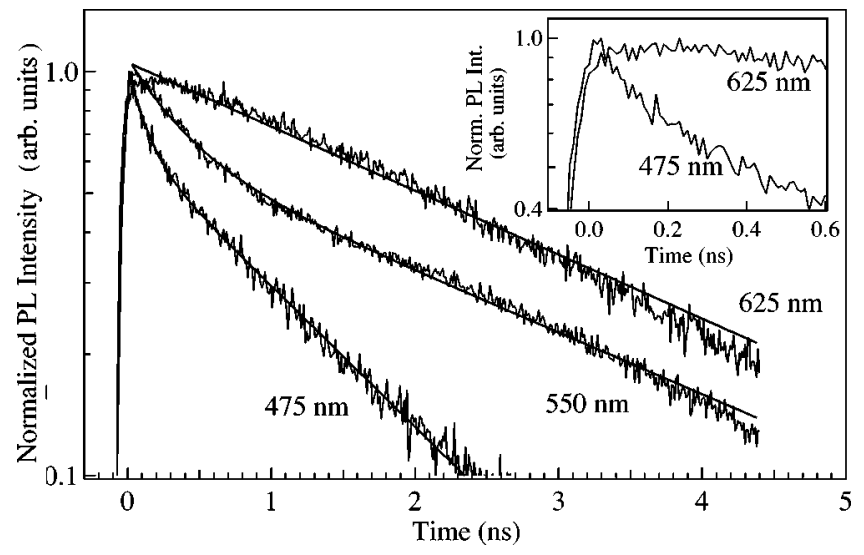

FIG. 3. The PL decay of PDPA- $n$ Bu film observed at different PL wavelengths of 475, 550, and $625 \mathrm{~nm}$.

be about $2.74 \mathrm{~ns}$. In contrast, the PL decay at shorter wavelengths is faster, and is better approximated by a doubleexponential decay:

$$
I(t)=I_{0 \mathrm{f}} \exp \left(-t / \tau_{\mathrm{f}}\right)+I_{0 \mathrm{~s}} \exp \left(-t / \tau_{\mathrm{s}}\right)
$$

where the decay components $\tau_{\mathrm{f}}$ and $\tau_{\mathrm{s}}$ are $0.37 \mathrm{~ns}$ and 2.84 ns for the PL decay at $550 \mathrm{~nm}$, and decrease to $0.1 \mathrm{~ns}$ and 1.2 $\mathrm{ns}$, respectively, for emission at $475 \mathrm{~nm}$.

Time-resolved PL spectra observed at different time between 0 ns and 3 ns after photoexcitation are shown in Fig. 4. A dynamic Stokes shift can be seen in these spectra, that is, the spectra are largely redshifted by about $20 \mathrm{~nm}$ (or about $0.1 \mathrm{eV}$ in photon energy) in less than $1 \mathrm{~ns}$ after photoexcitation, but the Stokes shift becomes much smaller at longer than $1 \mathrm{~ns}$. The observed PL spectra show a broad spectral shape of a single peak, which can be fitted by a single Gaussian spectral shape as a function of photon energy, but the PL spectrum observed within $0.5 \mathrm{~ns}$ after photoexcitation shows a small bump at short wavelengths, for example, around $475 \mathrm{~nm}$ at $0.1 \mathrm{~ns}$. This bump may be related to polymer chains having short effective conjugation lengths that exist due to an inhomogenous distribution of polymer chains with different effective average conjugation lengths. This result may be consistent with the reported observation of the sideband for both C-C single- and double-stretching

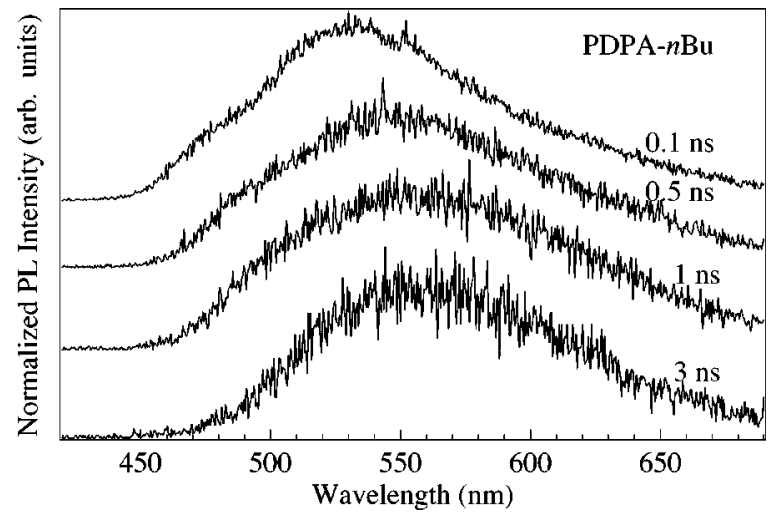

FIG. 4. The time-resolved PL spectra of PDPA- $n$ Bu film observed at different time, that is, $0.1,0.5,1$, and $3 \mathrm{~ns}$, after photoexcitation. 
peaks in the resonant Raman spectra of trans-polyacetylene, which is attributed to bimodal distribution of short and long segments. $^{25}$

The observed PL dynamics of pure PDPA- $n$ Bu can be interpreted as follows. Upon photoexcitation, the $1{ }^{1} B_{u}$ exciton is formed as a direct product of the intrachain excitation. This exciton may relax to a lower energy through lattice/ vibrational relaxation by the multiphonon process and then recombine radiatively as PL. This exciton can also be called a self-trapped (polaron) exciton, because a stabilized exciton of lower energy on a main chain cannot be easily transported to neighboring chains. The relaxation energy of about 0.4 $\mathrm{eV}$, which corresponds to a large Stokes shift, in PL of PDPA- $n \mathrm{Bu}$ is much larger than that of other conjugated polymers. This observation indicates that excitons in this polymer are strongly coupled to lattice/vibrational relaxation.

The PL spectrum observed at 0.1 ns shows a broader spectrum extending to shorter wavelengths compared with that observed at longer times. This may be interpreted according to the one-dimensional model of excitons diffusion among randomly distributed recombination centers. ${ }^{26}$ The portion of the spectrum at short wavelengths decay faster than that at longer wavelengths, since at short time after photoexcitation, the excitons in the short segments do not have to diffuse as far to reach recombination centers. However, at longer time, the survival excitons in the short segments may also relax to lower energy through migration to longer conjugation segments. These processes result in suppression of PL at short wavelengths as observed in PL spectrum at $3 \mathrm{~ns}$ in comparison with the spectrum at $0.1 \mathrm{~ns}$ in Fig. 4. This can be clearly seen in Fig. 3 as the short non-singleexponential PL decay observed in the short-wavelength range centered at $475 \mathrm{~nm}$. The PL decay at long wavelength $(625 \mathrm{~nm})$, however, shows a slow single-exponential decay because the exciton migration to longer conjugation length segment no longer occurs.

In general, the exciton migration may occur by exciton hopping from one segment to another segment either on the same chain or on neighboring chains, or through nonexcitonic processes such as long-range dipole-dipole interactions (Föster type of excitation energy transfer) or electron exchange interactions (Dexter mechanism). The redshift of PL spectra with time has also been observed in other polymers such as PPV and its derivatives, ${ }^{27}$ as well as in ladder-type poly(para-phenylene) (LPPP) ${ }^{28}$ In the case of PPV and its derivatives, the redshift has been explained by the exciton migration from short conjugation segment to the longer one, resulting in a fast non-single-exponential PL decay at short wavelengths, but a slow single-exponential decay at longer wavelengths because the excitons in a long conjugation segments cannot be transferred further. On the other hand, in LPPP, the redshift was explained by the transfer of excitation energy between two different emitting species, that is, from singlet exciton states to aggregate states.

It is well known, however, that in the polymers mentioned above the PL spectra are significantly different in solid film and solution. ${ }^{29,30}$ In contrast, we did not observe any large difference in PL spectra of PDPA derivatives in film and solution. The steady-state and time-resolved PL spectra of PDPA- $n \mathrm{Bu}$ films resemble to those of PDPA- $n \mathrm{Bu}$ in chloroform solution, indicating a weak interchain interaction.
Therefore, the possibility of PL redshift due to interchain specieses or aggregation could be excluded in PDPA- $n \mathrm{Bu}$ film. Since PDPA- $n$ Bu can be considered to have weak interchain interactions and strong exciton confinement on the main-chain segments, we suggests that exciton migration through long-range interactions such as dipole-dipole interactions (Förster mechanism) is more probable than other mechanisms.

In contrast to the case of the PDPA- $n \mathrm{Bu}$ solid film, PL decay in PDPA- $n \mathrm{Bu}$ solution is almost independent of emission wavelength, that is, it is a single-exponential decay with a lifetime constant of about 0.9 ns. The redshift in timeresolved PL spectra is not so obvious in solution. These differences between PL in the thin film and solution could be explained by the faster molecular relaxation process on a single chain in solution rather than in solid film. However, similar PL decay characteristics to that observed in solution were also observed in the solid film of the PDPA- $n$ Bu/polystyrene blend, implying that the PL decay characteristics in solution and polymer blend should be originated from excitons confined in single chains. That is, because of the large distance of interchain separation, interchain exciton migration becomes improbable.

Exciton migration from the short conjugation segments to the long conjugation segments can take place up to a few hundred picoseconds after photoexcitation, as indicated in the inset of Fig. 3. That is, as shown in this figure, PL intensity at the wavelength of $625 \mathrm{~nm}$ increases slightly up to 100 ps after the excitation and then slowly decreases. Curve fitting of the experimental results in Fig. 3 with Eq. (2) indicates that the lifetimes of the slow PL decay component $\left(\tau_{\mathrm{s}}\right)$ at wavelengths of $550 \mathrm{~nm}$ and $625 \mathrm{~nm}$ are almost the same, about $2.8 \mathrm{~ns}$, but $\tau_{\mathrm{s}}$ at the wavelength of $475 \mathrm{~nm}$ is shorter (about $1.2 \mathrm{~ns}$ ). The lifetime of the fast PL decay component $\left(\tau_{\mathrm{f}}\right)$ at $475 \mathrm{~nm}$ is also shorter than that at $550 \mathrm{~nm}$, that is, about $0.37 \mathrm{~ns}$ at $550 \mathrm{~nm}$ but $0.1 \mathrm{~ns}$ at $475 \mathrm{~nm}$. This indicates that the decay dynamics of excitons in the short segments is faster than in the long segments and should be determined by the competitive process between radiative recombination and exciton migration to long conjugation segments.

In order to study further the exciton dynamics in this polymer, we studied the polymer blend of highly luminescent PDPA- $n \mathrm{Bu}$ and poorly luminescent PPA- $o \mathrm{SiMe}_{3}$. Figures 5(a), 5(b) and 5(c) show normalized PL decay of the PDPA- $n$ Bu/PPA- $o \mathrm{SiMe}_{3}$ blend films with various molar ratios observed at PL wavelengths of 475, 550, and $625 \mathrm{~nm}$, respectively. Figure 6 shows the corresponding timeresolved PL spectra of these blend films with various molar ratios observed until $0.2 \mathrm{~ns}$ after photoexcitation. The PL spectra indicate that PL originates only from PDPA- $n \mathrm{Bu}$ without any contribution from PPA-o $\mathrm{SiMe}_{3}$. The PL decay in Fig. 5 indicates that the PL lifetime is drastically shortened by mixing PPA- $o \mathrm{SiMe}_{3}$ in PDPA- $n \mathrm{Bu}$.

For all molar ratios of the PDPA- $n \mathrm{Bu} / \mathrm{PPA}-o \mathrm{SiMe}_{3}$ polymer blends the PL decay at the wavelength of $625 \mathrm{~nm}$ can be approximated by a single-exponential decay function but cannot for the PL decay at shorter wavelengths, which should be related to the PL dynamics of PDPA- $n \mathrm{Bu}$ itself. That is, the PL decay at shorter wavelengths must be fitted by a double-exponential function, as we did for PL decay of pure PDPA- $n \mathrm{Bu}$. The lifetime of PL decay at the wavelength 


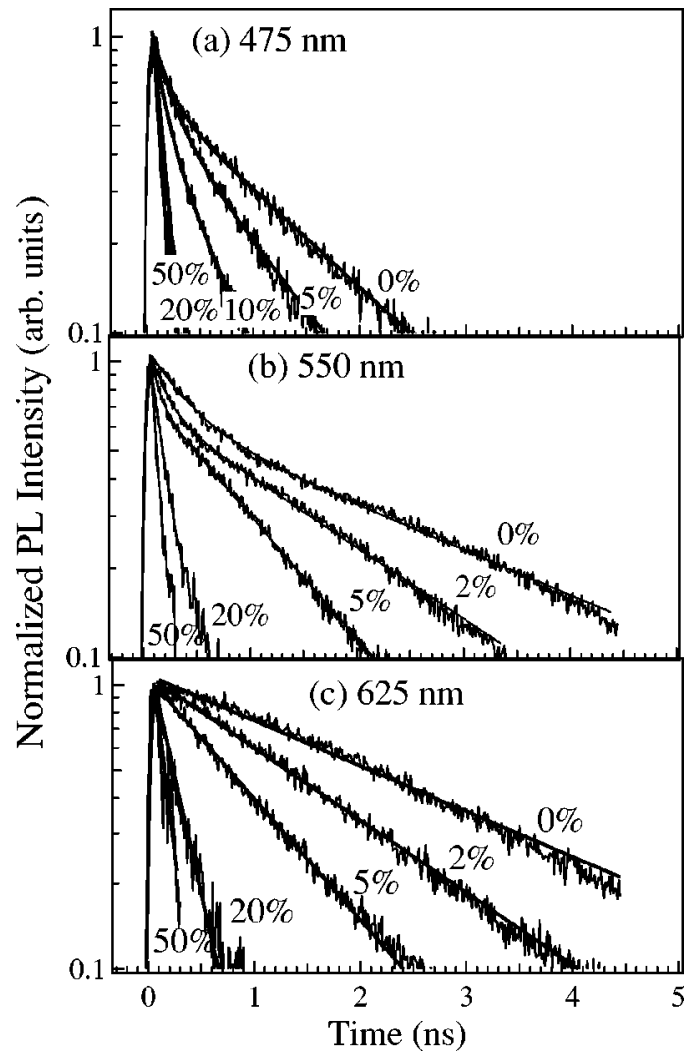

FIG. 5. The PL decay of PDPA- $n \mathrm{Bu} / \mathrm{PPA}-o \mathrm{SiMe}_{3}$ blends dependent on molar ratio observed at different PL wavelengths of (a) $475 \mathrm{~nm}$, (b) $550 \mathrm{~nm}$, and (c) $625 \mathrm{~nm}$.

of $625 \mathrm{~nm}$ rapidly decreases with increase of the molar ratio of PPA-oSiMe 3 , as shown in Fig. 7. This result suggests exciton migration from PDPA- $n$ Bu with a large band gap to PPA-oSiMe ${ }_{3}$ with a small band gap, which may occur through Förster excitation energy transfer, ${ }^{31}$ by considering the exciton dynamics in pure PDPA- $n$ Bu. However, further experiments are required for clarification of the process. The spectral overlap between the PL spectrum of a donor molecule and absorption spectrum of an acceptor molecule required for Förster energy transfer is fulfilled since the PL of PDPA- $n \mathrm{Bu}$ (peak at $2.3 \mathrm{eV}$ ) is higher in energy than the energy gap of PPA-o $\mathrm{SiMe}_{3}(1.9 \mathrm{eV})$, as shown in Fig. 2. The

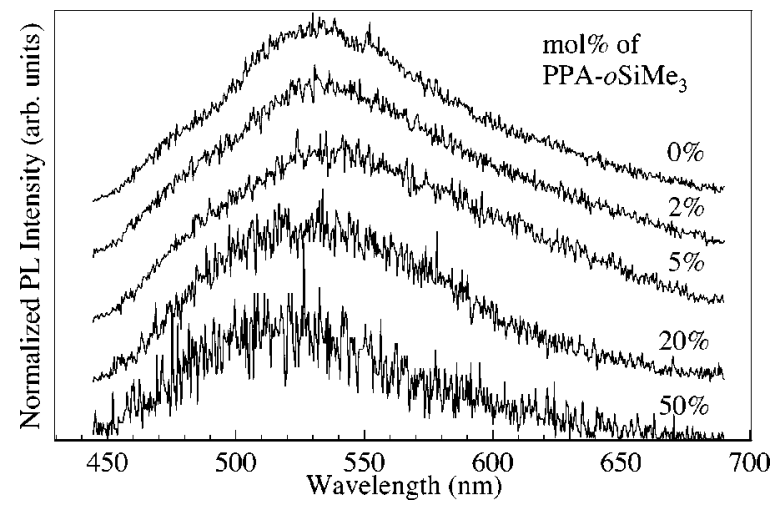

FIG. 6. The time-resolved PL spectra of PDPA- $n \mathrm{Bu} /$ PPA- $o \mathrm{SiMe}_{3}$ blends dependent on molar ratio observed at $0.1 \mathrm{~ns}$ after photoexcitation.

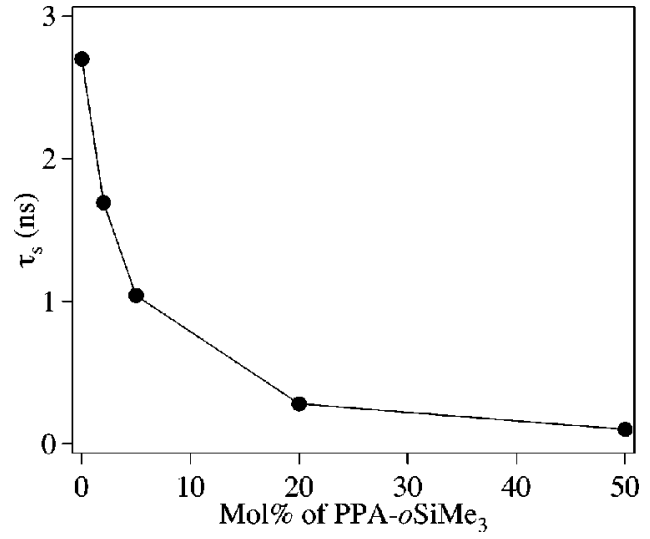

FIG. 7. The decreasing of PL lifetime at PL wavelength of 625 $\mathrm{nm}$ in polymer blend PDPA- $n \mathrm{Bu} / \mathrm{PPA}-o \mathrm{SiMe}_{3}$ with increasing molar ratio of PPA-oSiMe

occurrence of excitation energy transfer in conjugated polymer blends has recently been reported such as in a blend of PPPV [poly(phenylphenylenevinylene)] with dye molecules $^{32,33}$ and also in a blend of LPPP and poly(perylene-co-diethynylbenzene) (PPDB). ${ }^{34,35}$ The observation of PL quenching in PDPA- $n$ Bu due to Förster energy transfer to PPA-o $\mathrm{SiMe}_{3}$ may support the interpretation that $\mathrm{PL}$ is originated from excitons, but not from recombination of separated free charge carriers or polarons.

In general, Förster type of excitation energy transfer between two molecules may occur through dipole-dipole resonance when both molecules have an allowed dipole transition and through dipole-quadrapole resonance when one of the molecules has a forbidden dipole transition, with transfer rates proportional to $R^{-6}$ for dipole-dipole resonance and $R^{-8}$ for dipole-quadrapole resonance ( $R$ is the distance between the molecules). Therefore, this process may lead PPA- $o \mathrm{SiMe}_{3}$ to either $1{ }^{1} B_{u}$ or $2{ }^{1} A_{g}$ excited states. In both cases, the excited PPA-o $\mathrm{SiMe}_{3}$ may relax nonradiatively to the ground state because its $2{ }^{1} A_{g}$ excited state lies below the $1{ }^{1} B_{u}$ excited state in energy. ${ }^{13,14}$ However, it is also possible that the $2{ }^{1} A_{g}$ excited state relaxes into soliton pairs in a very short time. Interpretation in terms of the nonradiative decay of excitons in PPA-o $\mathrm{SiMe}_{3}$ with phonon emission in a few picoseconds is consistent with the results in a recent report of redshift of the optical absorption spectrum in the timeresolved photomodulation measurements. ${ }^{36}$

As shown in Fig. 6, the PL peak in the 50\%:50\% polymer blend weakly appears at shorter wavelengths in comparison with that in the pristine PDPA- $n \mathrm{Bu}$ sample. This could indicate that in the 50\%:50\% polymer blend each PDPA- $n \mathrm{Bu}$ chain may be surrounded by PPA-o $\mathrm{SiMe}_{3}$ chains. In such environment, PDPA- $n$ Bu chains may have a little different molecular conformation, leading to a shorter effective average conjugation length and modification of the energy of the self-trapped (polaron) exciton in PDPA- $n \mathrm{Bu}$ to slightly higher energy. That is, in the polymer blend of PDPA- $n$ Bu:PPA- $o \mathrm{SiMe}_{3}=80 \%: 20 \%$ there still exist some PDPA- $n \mathrm{Bu}$ chains that are free from the influence of PPA- $O \mathrm{SiMe}_{3}$, but in the 50\%:50\% polymer blend most PDPA- $n$ Bu chains have nearby PPA- $o \mathrm{SiMe}_{3}$ chains, resulting in the shift of PL spectra to shorter wavelengths. 
Alternatively, the observation of the PL peak at shorter wavelengths in the 50\%:50\% polymer blend than in the pristine sample could also be explained by considering the competitive processes between the exciton migration in PDPA- $n \mathrm{Bu}$ chain segments with different conjugation lengths and exciton migration from PDPA- $n \mathrm{Bu}$ to PPA- $o \mathrm{SiMe}_{3}$. That is, in the presence of PPA-oSiMe tons in short conjugation segments of PDPA- $n$ Bu may more easily migrate to PPA-oSiMe 3 rather than migrate to other PDPA- $n \mathrm{Bu}$ segments with longer conjugation. Therefore, with increasing molar ratio of PPA-o $\mathrm{SiMe}_{3}$, the redshift of $\mathrm{PL}$ spectra due to exciton migration in PDPA- $n \mathrm{Bu}$ is no longer observed. Instead, the appearance of PL spectra peaking at shorter wavelength was observed due to the surviving excitons in short conjugation segments. This may be consistent with the fact that excited PDPA- $n$ Bu are in a good resonance condition with PPA-o $\mathrm{SiMe}_{3}$, which can be seen from the spectral overlap of PL of PDPA- $n \mathrm{Bu}$ and absorption of PPA- $o \mathrm{SiMe}_{3}$ in Fig. 2, but the resonance might be better at longer wavelengths. It should also be noted that even at 0.1 ns after photoexcitation the PL spectrum of the blend with PPA- $o \mathrm{SiMe}_{3}$ of $50 \%$ is narrower than that of the purePDPA- $n \mathrm{Bu}$. This may suggest that the exciton migration from PDPA- $n \mathrm{Bu}$ to PPA- $o \mathrm{SiMe}_{3}$ takes place in less than 0.1 ns. This suggestion may be plausible, since for comparison, the charge transfer in blends of poly(3-octylthiophene) (P3OT) $/ \mathrm{C}_{60}$ occurs within less than $1 \mathrm{ps}$, resulting in PL quenching and photoinduced charge transfer. ${ }^{37}$

The exciton migration discussed here may explain the observation of the drastic PL spectral shift to green luminescence upon mixing a small amount of PDPA- $n \mathrm{Bu}$ with the blue luminescent PAPA and the observation of stronger electroluminescence originating from PPA- $\mathrm{SiMe}_{3}$ in the polymer blend of PDPA- $n$ Bu/PPA- $o \mathrm{SiMe}_{3}$ than in pure PPA- $o \mathrm{SiMe}_{3} \cdot{ }^{38,21}$

\section{SUMMARY}

We have observed different PL decay characteristics depending on the wavelength of the emission and the dynamic Stokes shift of time-resolved PL spectra in pure-PDPA- $n$ Bu film. The PL is interpreted to originate from the radiative decay of self-trapped (polaron) excitons, that is, photogenerated $1{ }^{1} B_{u}$ excitons, which are stabilized to a lower energy by lattice/vibrational relaxation, as indicated by a large Stokes shift of the PL spectrum. The photoexcitation of short conjugation segments results in radiative recombination of excitons in competition with exciton migration to longer conjugation segments, resulting in fast decay of shortwavelength PL and redshift of time-resolved PL spectra with time.

In the polymer blends of PDPA- $n \mathrm{Bu} / \mathrm{PPA}-o \mathrm{SiMe}_{3}$, the time-resolved PL spectra show that PL originates only from PDPA- $n$ Bu for all molar ratios, but the lifetime drastically decreases upon mixing PDPA- $n \mathrm{Bu}$ with a small amount of PPA-oSiMe ${ }_{3}$. With increasing molar ratio of PPA-oSiMe the long-wavelength PL was more weakened than the shortwavelength PL, and the decrease of lifetime is more marked in the long-wavelength PL than the short-wavelength PL. The result could be interpreted in terms of exciton migration, which may occur through Förster energy transfer, from PDPA- $n$ Bu to PPA- $o \mathrm{SiMe}_{3}$ with a much faster rate than exciton migration to longer conjugation segments of PDPA- $n$ Bu. However, it should also be noted that our present results cannot completely exclude the possibility of the charge-transfer process, that is, both energy transfer and charge transfer might be possible.

\section{ACKNOWLEDGMENTS}

Part of this work was financially supported by the Research for the Future Program of the Japan Society for the Promotion of Science (Project No. JSPS-RFTF96P00206).
${ }^{1}$ A. J. Heeger, S. Kivelson, J. R. Schrieffer, and W. P. Su, Rev. Mod. Phys. 60, 781 (1988).

${ }^{2}$ J. H. Burroughes, D. D. C. Bradley, A. R. Brown, R. N. Marks, K. Mackey, R. H. Friend, P. L. Burns, and A. B. Holmes, Nature (London) 347, 539 (1990).

${ }^{3}$ Y. Ohmori, M. Uchida, K. Muro, and K. Yoshino, Jpn. J. Appl. Phys., Part 2 30, L1938 (1991).

${ }^{4}$ D. Braun and A. J. Heeger, Appl. Phys. Lett. 58, 1982 (1991).

${ }^{5}$ S. Heun, R. F. Mahrt, A. Greiner, U. Lemmer, H. Bässler, D. A. Halliday, D. D. C. Bradley, P. L. Burn, and A. B. Holmes, J. Phys.: Condens. Matter 5, 247 (1993).

${ }^{6}$ R. Kersting, U. Lemmer, R. F. Mahrt, K. Leo, H. Kurz, H. Bässler, and E. O. Göbel, Phys. Rev. Lett. 24, 3820 (1993).

${ }^{7}$ M. Yan, L. J. Rothberg, E. W. Kock, and T. M. Miller, Phys. Rev. Lett. 75, 1992 (1995).

${ }^{8}$ I. D. W. Samuel, G. Rumbles, and C. J. Collison, Phys. Rev. B 52, 11573 (1995)

${ }^{9}$ U. Lemmer, S. Heun, R. F. Mahrt, U. Scherf, M. Hopmeier, U. Siegner, E. O. Göbel, K. Mullen, and H. Bässler, Chem. Phys. Lett. 240, 373 (1995).

${ }^{10}$ L. Lauchlan, S. Etemad, T. C. Chung, A. J. Heeger, and A. G. MacDiarmid, Phys. Rev. B 24, 3701 (1981).
${ }^{11}$ K. Yoshino, S. Hayashi, Y. Inuishi, K. Hattori, and Y. Watanabe, Solid State Commun. 46, 583 (1983).

${ }^{12}$ D. B. Fitchen, Synth. Met. 9, 341 (1984).

${ }^{13}$ G. S. Kanner, S. V. Frolov, and Z. V. Vardeny, Phys. Rev. Lett. 74, 1685 (1995).

${ }^{14}$ M. Liess, S. Jeglinski, Z. V. Vardeny, M. Ozaki, K. Yoshino, Y. Ding, and T. Barton, Phys. Rev. B 56, 15712 (1997).

${ }^{15}$ P. Tavan and K. Schulten, Phys. Rev. B 36, 4337 (1987).

${ }^{16}$ R. J. Bursill and W. Barford, Phys. Rev. Lett. 82, 1514 (1999).

${ }^{17}$ K. Tada, R. Hidayat, M. Hirohata, M. Teraguchi, T. Masuda, and K. Yoshino, Jpn. J. Appl. Phys., Part 2 35, L1138 (1996).

${ }^{18}$ I. Gontia, S. V. Frolov, M. Liess, E. Ehrenfreund, Z. V. Vardeny, K. Tada, H. Kajii, R. Hidayat, A. Fujii, K. Yoshino, M. Teraguchi, and T. Masuda, Phys. Rev. Lett. 82, 4058 (1999).

${ }^{19}$ M. Hirohata, K. Tada, R. Hidayat, T. Masuda, and K. Yoshino, Jpn. J. Appl. Phys., Part 2 36, L302 (1997).

${ }^{20}$ R. Hidayat, M. Hirohata, K. Tada, M. Teraguchi, T. Masuda, and K. Yoshino, Jpn. J. Appl. Phys., Part 1 36, 3740 (1997).

${ }^{21}$ R. Hidayat, M. Hirohata, A. Fujii, M. Teraguchi, T. Masuda, and K. Yoshino, Jpn. J. Appl. Phys., Part 1 38, 4726 (1999).

${ }^{22}$ T. Masuda, T. Hamano, K. Tsuchihara, and T. Higashimura, Macromolecules 23, 1374 (1990). 
${ }^{23}$ H. Shirakawa, T. Masuda, and K. Takeda, in The Chemistry of Triple-Bonded Functional Groups, edited by S. Patai (Wiley, New York, 1994).

${ }^{24}$ N. C. Greenham, I. D. W. Samuel, G. R. Hayes, R. T. Phillips, Y. A. R. R. Kessener, S. C. Moratti, A. B. Holmes, and R. H. Friend, Chem. Phys. Lett. 241, 89 (1995).

${ }^{25}$ G. P. Brivio and E. Mulazzi, Phys. Rev. B 30, 876 (1984).

${ }^{26}$ G. S. Kanner and Z. V. Vardeny, Synth. Met. 50, 611 (1992).

${ }^{27}$ G. R. Hayes, I. D. W. Samuel, and R. T. Phillips, Phys. Rev. B 52, R11 569 (1995).

${ }^{28}$ G. Kranzelbinder, H. J. Byrne, S. Hallstein, S. Roth, G. Leising, and U. Scherf, Phys. Rev. B 56, 1632 (1997).

${ }^{29}$ I. D. W. Samuel, G. Rumbles, C. J. Collison, R. H. Friend, S. C. Moratti, and A. B. Holmes, Synth. Met. 84, 497 (1997).

${ }^{30}$ U. Lemmer, S. Heun, R. F. Mahrt, U. Scherf, M. Hopmeier, U. Siegner, E. O. Göbel, K. Mullen, and H. Bässler, Chem. Phys. Lett. 240, 373 (1995).

${ }^{31}$ F. Gutmann, H. Keyzer, L. E. Lyons, and R. B. Somano, Organic Semiconductors-Part B (Krieger, Malabar, 1983), Chap. 5, p. 273.
${ }^{32}$ U. Lemmer, A. Ochse, M. Deussen, R. F. Mahrt, E. O. Göbel, H. Bässler, P. Haring Bolivar, G. Wegmann, and H. Kurz, Synth. Met. 78, 289 (1996).

${ }^{33}$ P. Haring Bolivar, G. Wegmann, R. Kersting, M. Deussen, U. Lemmer, R. F. Mahrt, H. Bässler, E. O. Göbel, and H. Kurz, Chem. Phys. Lett. 245, 534 (1995).

${ }^{34}$ G. Cerullo, M. Nisoli, S. Stagira, S. De Silvestri, G. Lanzani, W. Graupner, E. J. W. List, and G. Leising, Chem. Phys. Lett. 288, 561 (1998).

${ }^{35}$ M. Wohlgenannt, W. Graupner, F. P. Wenzl, S. Tasch, E. J. W. List, G. Leising, M. Graupner, A. Hermetter, U. Rohr, P. Schlichting, Y. Geerts, U. Scherf, and K. Mullen, Chem. Phys. 227, 99 (1998).

${ }^{36}$ J. D. Huang, S. V. Frolov, Z. V. Vardeny, W. Chen, T. J. Barton, R. Sugimoto, M. Ozaki, and K. Yoshino, Proc. SPIE 3145, 495 (1997).

${ }^{37}$ B. Kraabel, C. H. Lee, D. McBranch, D. Moses, N. S. Sariciftci, and A. J. Heeger, Chem. Phys. Lett. 213, 389 (1993).

${ }^{38}$ R. Hidayat, M. Hirohata, K. Tada, M. Teraguchi, T. Masuda, and K. Yoshino, Jpn. J. Appl. Phys., Part 2 37, L180 (1998). 